\title{
HOLONOMIC MODULES FOR RINGS OF INVARIANT DIFFERENTIAL OPERATORS
}

\author{
VYACHESLAV FUTORNY AND JOÃO SCHWARZ
}

\begin{abstract}
We study holonomic modules for the rings of invariant differential operators on affine commutative domains with finite Krull dimension with respect to arbitrary actions of finite groups. We prove the Bernstein inequality for these rings. Our main tool is the filter dimension introduced by Bavula. We extend the results for the invariants of the Weyl algebra with respect to the symplectic action of a finite group, for the rings of invariant differential operators on quotient varieties, and invariants of certain generalized Weyl algebras under the linear actions. We show that the filter dimension of all above mentioned algebras equals 1 .
\end{abstract}

Keywords: Filter dimension, holonomic modules, generalized Weyl algebras, invariant differential operators

2020 Mathematics Subject Classification: 16P90, 16S32, 16D30, $16 \mathrm{~W} 70$

\section{INTRODUCTION}

Let $k$ be the base field. All rings in the paper are k-algebras. All modules are left modules, unless said otherwise.

In this paper we address representations of subrings of invariants of generalized Weyl algebras. The later class of algebras was introduced and studied in [2]. Many important algebras of small Gelfand-Kirillov dimension arising in noncommutative geometry are generalized Weyl algebras, such as the first Weyl algebra and its quantization; the quantum plane and the quantum sphere; $U\left(s l_{2}(\mathrm{k})\right)$ and its quantization; the Heisenberg algebra and its quantizations; quantum $2 \times 2$ matrices; Witten's and Woronowic's deformations; Noetherian down-up algebras (cf. 9]). For the representation theory of generalized Weyl algebras we refer to [2, 4], [5], [11, [12.

We will consider a category of holonomic modules for certain subrings of invariants of generalized Weyl algebras under the action of finite groups.

For an arbitrary algebra $A$ denote by $G K(A)$ the Gelfand-Kirillov dimension of $A$. Then a finitely generated $A$-module $M$ is called holonomic (cf. 20, 24]) if

$$
G K(M)=\frac{1}{2} G K(A / A n n(M)) .
$$

Assume $\operatorname{char} k=0$ and consider the $n$-th Weyl algebra $A_{n}(\mathrm{k})$. Then $A_{n}(\mathrm{k})$ is isomorphic to the ring of differential operators on the polynomial algebra $\mathrm{k}\left[x_{1}, \ldots, x_{n}\right]$, or equivalently on the affine space $\mathbb{A}^{n}$. It has generators $x_{1}, \ldots, x_{n}, y_{1}, \ldots, y_{n}$ and defining relations

$$
\left[x_{i}, x_{j}\right]=\left[y_{i}, y_{j}\right]=0 ;\left[y_{i}, x_{j}\right]=\delta_{i j}
$$


$i, j=1, \ldots, n$. Note that $G K\left(A_{n}(\mathrm{k})\right)=2 n$ and for every finitely generated $A_{n}(\mathrm{k})$ module $M$ holds the Bernstein inequality: $G K(M) \geq n$ [15. Holonomic $A_{n}(\mathrm{k})$ modules are exactly the modules of the minimal Gelfand-Kirillov dimension, they constitute an important abelian subcategory of modules for the Weyl algebra ([16]). The Bernstein inequality holds also for the rings of differential operators on smooth affine varieties ([16]; cf. Section 3 bellow).

Assume $k$ algebraically closed of characteristic 0 and let $\mathfrak{g}$ be an algebraic Lie algebra of finite dimension. Then the Gabber's inequality

$$
G K(U(\mathfrak{g}) / \operatorname{Ann}(M)) \leq 2 G K(M)
$$

holds for any finitely generated $\mathfrak{g}$-module $M$ (cf. [19]). The holonomic $\mathfrak{g}$-modules are those modules with the minimal Gelfand-Kirillov dimension (cf. 20, Chapter 9). However, if $\mathfrak{g}$ is not algebraic then it is known that the Gabber's inequality does not hold 23 .

To study holonomic modules and analogues of the Bernstein inequality for infinitedimensional affine simple algebras over an arbitrary field, Bavula 4 introduced the notion of the filter dimension, denoted here by fdim (see also [8] for details and applications). Our first main result gives the filter dimension of certain algebras of invariant differential operators with respect to finite groups action.

Theorem 1.1. The filter dimension equals 1 for the following algebras:

- $\mathcal{D}(A)^{G}$, where $A$ is an affine regular commutative domain with the finite Krull dimension and $G$ is a finite group of automorphisms of $A$;

- $A_{n}(\mathrm{k})^{G}$, where $G$ a finite group of symplectic automorphisms of the Weyl algebra $A_{n}(\mathrm{k})$;

- $\mathcal{D}\left(\mathbb{A}^{n} / G\right), \mathrm{k}$ is algebraically closed and $G$ is a finite group of linear automorphisms of the affine space $\mathbb{A}_{\mathrm{k}}^{n}$.

We also extend the results of [10] for the invariants of generalized Weyl algebras under suitable actions of complex reflection groups of type $G(m, p, r)$. Namely, we have

Theorem 1.2. Let $D(a, \sigma)$ be a generalized Weyl algebra rank $r$ of pure type and $G=G(m, p, r)$. Then $f \operatorname{dim} D(a, \sigma)^{G}=1$.

With the idea of the filter dimension we have an alternative definition of holonomic modules.

Definition 1.3. Let $A$ be an affine infinite-dimensional simple algebra. The infimum $h_{A}$ of the set

$$
\{G K(M) \mid M \text { is finitely generated } A \text {-module }\}
$$

is called the holonomic number of $A$.

Suppose that $A$ is simple, $f \operatorname{dim} A \geq 1$ and the infimum $h_{A}$ in Definition 1.3 is actually the minimum. Then in this case the definition of a holonomic $A$-module above can be replaced by the following: a finitely generated $A$-module $M$ is holonomic if $G K(M)=h_{A}$ (cf. Theorem 2.3). The situation though is unclear if $\operatorname{fdim} A<1$.

It was shown in 10 that, given a finite Coxeter group $W$ action on the Weyl algebra $A_{n}(\mathrm{k})$, the Bernstein inequality holds for $A_{n}(\mathrm{k})^{W}$ : for every finitely generated $A_{n}(\mathrm{k})^{W}$-module $M, G K(M) \geq n$. We generalize this result and prove the 
Bernstein inequality for $A_{n}(\mathrm{k})^{G}$ with linear action of an arbitrary finite group $G$. We note that our approach is different from the one in [10] (cf. Theorem 5.7 bellow). We also prove a similar result for more general actions of finite groups of symplectic automorphisms on $A_{n}(\mathrm{k})$. Moreover, we extend this result to the ring of invariant differential operators $\mathcal{D}(A)^{G}$ on an arbitrary affine regular commutative domain $A$ with finite Krull dimension and any finite group $G$. Our approach relies on the computation of the filter dimension in order to illustrate its application. In particular, all algebras mentioned in Theorem 1.1 and Theorem 1.2 have a "good" theory of holonomic modules:

Theorem 1.4. Let $A$ be an affine regular commutative domain with finite Krull dimension and $G K(A)=n$, and let $B$ be one of the algebras from Theorem 1.1 or Theorem 1.2. Then every finitely generated holonomic B-module is a cyclic torsion module of finite length and their holonomic number is $n$.

We apply developed technique to compute the filtered dimension and the Krull dimension of rational Cherednik algebras. In particular, we show

Theorem 1.5. The filtered dimension of generic rational Cherednik algebras and their spherical subalgebras equals 1.

The structure of the paper is as follows. In Section 2 we recall the notion of a filter dimension and its main properties. Section 3 is the technical core of the paper: we prove a number of results on holonomic modules for simple somewhat commutative algebras, and simple filtered semi-commutative algebras which are Noetherian but not Artinian, with particular emphasis on certain generalized Weyl algebras. All of these algebras are examples of so-called algebras with multiplicity. Some of these results are probably known to specialists but we included them for the clarity of exposition. In Section 4 we discuss the filter dimension of rings of invariants under the action of a finite group. In Section 5 we consider the invariants of Weyl algebras, rings of invariant differential operators on quotient varieties, and invariants of generalized Weyl algebras. We show that these algebras have a nice category of holonomic modules (cf. Theorems 5.7, 5.8, 5.11, 5.9). Finally, in Section 6 we compute the filtered dimension and the Krull dimension of the rational Cherednik algebras and its spherical subalgebras.

\section{The Filter Dimension}

Let $A$ be an algebra over k. Every finite-dimensional subspace $V \subset A$ containing the identity will be called a frame of $A$. Let us recall the notion of the GelfandKirillov (GK) dimension of algebras and modules (cf. 20]).

\section{Definition 2.1.}

$$
G K(A)=\sup _{V} \limsup _{n \mapsto \infty} \frac{\log \operatorname{dim} V^{n}}{\log n},
$$

where $V$ ranges through all frames of $A$. Let $M$ be an A-module. Then

$$
G K(M)=\sup _{V, F} \limsup _{n \mapsto \infty} \frac{\log \operatorname{dim} V^{n} F}{\log n}
$$

where $F$ runs through all finite dimensional subspaces of $M$ and $V$ runs through all frames of $A$. 
Recall ([24, 8.1.9]) that a finite-dimensional filtration of an algebra $A$ is a filtration $\mathcal{F}=\left\{A_{i}\right\}_{i \geq 0}$ such that $A_{0}=\mathrm{k}$ and $\operatorname{dim} A_{i}<\infty, i>0$. If $N$ is an $A$-module with filtration $\left\{N_{i}\right\}_{i \geq 0}$, then the filtration is finite-dimensional if $\operatorname{dim} N_{i}<\infty$, $i \geq 0$. Suppose that $A$ is an infinite-dimensional affine algebra generated by $a_{1}, \ldots a_{n}$. Define a finite-dimensional filtration $\mathcal{F}=\left\{A_{i}\right\}_{i \geq 0}$ in the following way: $A_{0}=\mathrm{k}, A_{1}=\operatorname{span}\left\langle 1, a_{1}, \ldots, a_{n}\right\rangle, A_{i}=A_{1}^{i}$. Now let $M=A M_{0}$ be a finitely generated $A$-module with finite-dimensional generating space $M_{0}$. Then $\mathcal{F}$ induces a natural filtration $\Omega=\left\{M_{i}\right\}_{i \geq 0}$ on $M$, where $M_{i}=A_{i} M_{0}$.

The return function $f_{\mathcal{F}, M_{0}}: \mathbb{N} \rightarrow \mathbb{N} \cup\{\infty\}$ is defined as follows: $f_{\mathcal{F}, M_{0}}(i)=$

$$
\min \left\{j \in \mathbb{N} \cup\{\infty\}: A_{j} M_{i, g} \supset M_{0} \forall M_{i, g}\right\},
$$

where $M_{i, g} \subset M_{i}$ runs over the finite-dimensional subspaces which generate $M$ as a module.

For each function $f: \mathbb{N} \rightarrow \mathbb{N} \cup\{\infty\}$ define $\Gamma(f)$ as

$$
\text { inf }\left\{r \in \mathbb{R} \mid f(i) \leq i^{r}, i>>0\right\} .
$$

Note that, if $f(i)=p(i)$ for all sufficiently large $i$ for some polynomial $p \in \mathbb{Q}[x]$, then $\Gamma(f)=\operatorname{deg} p(20$, Chp. 2).

For a finitely generated $A$-module $M$, the filter dimension is defined as ([4])

$$
f \operatorname{dim}(M)=\Gamma\left(f_{\mathcal{F}, M_{0}}\right) .
$$

It is independent of the choice of the generating set $a_{1}, \ldots, a_{n}$ and the subspace $M_{0}$ (4, Lemma 1.1]). The filter dimension of $A, f \operatorname{dim}(A)$, is its filter dimension as an $A \otimes_{\mathrm{k}} A^{o p}$-module.

Example 2.2 ([6]). Let $B$ be an affine regular commutative algebra with finite Krull dimension. Then the filter dimension of the ring of differential operators $\mathcal{D}(B)$ on $B$ equals 1.

The following analog of the Bernstein inequality was shown in 4]:

Theorem 2.3. Let $A$ be an infinite-dimensional affine simple algebra and $M a$ non-zero finitely generated module. Then

$$
G K(M) \geq \frac{G K(A)}{1+\max (1+f \operatorname{dim}(A))} .
$$

In view of Example 2.2, this reproves the usual Bernstein inequality for rings of differential operators on smooth affine varieties (cf. [16]).

In general, a computation of the filter dimension of an algebra is a difficult problem ([8], 20] 12.9). However, it has an important property to be Morita invariant. Namely:

Theorem 2.4 ([10], Theorem 1.3, Theorem 1.6). Morita equivalent infinite-dimensional affine simple algebras have the same filter dimension and the same holonomic number.

\section{Holonomic modules for algebras With multiplicity}

3.1. Algebras with multiplicity. From now on we assume that $\operatorname{char} \mathrm{k}=0$. Let $A$ be a finitely generated infinite-dimensional simple Noetherian algebra, with a finitedimensional filtration $\mathcal{F}=\left\{A_{i}\right\}_{i \geq 0}$ such that $g r_{\mathcal{F}} A$ is finitely generated Noetherian. 
Let $M$ be a finitely generated module over $A$ with a filtration $\Omega=\left\{M_{i}\right\}_{i \geq 0}$. We assume that $\Omega$ is a good filtration, that is $g r_{\Omega} M$ is a finitely generated module over $g r_{\mathcal{F}} A$. Recall that every finitely generated module has a good filtration ([20], Ch. $6)$.

Consider a short exact sequence of modules $0 \rightarrow M^{\prime} \rightarrow M \rightarrow M^{\prime \prime} \rightarrow 0$ and the filtrations $\Omega^{\prime}=\left\{M_{i}^{\prime}=M_{i} \cap M^{\prime}\right\}_{i \geq 0}$ and $\Omega^{\prime \prime}=\left\{M_{i}^{\prime \prime}=M_{i}+M / M\right\}_{i \geq 0}$ of $M^{\prime}$ and $M^{\prime \prime}$ respectively. This induces an exact sequence of $g r_{\mathcal{F}} A$-modules:

$$
0 \rightarrow g r_{\Omega^{\prime}} M^{\prime} \rightarrow g r_{\Omega} M \rightarrow g r_{\Omega^{\prime \prime}} M^{\prime \prime} \rightarrow 0 .
$$

Since $g r_{\Omega} M$ is finitely generated and $g r_{\mathcal{F}} A$ is Noetherian then $\Omega^{\prime}$ and $\Omega^{\prime \prime}$ are good filtrations.

Definition 3.1. [20, 12.6] We will say that $A$ is an algebra with multiplicity if for every finitely generated A-module $0 \neq M, G K(M)$ is a non-negative integer, and there is a function $M \mapsto e(M)$, where $e(M)$ is a non-negative integer, called the multiplicity of $M$ such that: for a short exact sequence of finitely generated A-modules

$$
0 \rightarrow M^{\prime} \rightarrow M \rightarrow M^{\prime \prime} \rightarrow 0
$$

we have $G K(M)=\max \left\{G K\left(M^{\prime}\right), G K\left(M^{\prime \prime}\right)\right\}$, and if $G K(M)=G K\left(M^{\prime}\right)=$ $G K\left(M^{\prime \prime}\right)$, then $e(M)=e\left(M^{\prime}\right)+e\left(M^{\prime \prime}\right)$.

Since the Gelfand-Kirillov dimension of finitely generated modules are nonnegative integers, there exists a non-zero module whose Gelfand-Kirillov dimension is precisely the holonomic number, which is a non-negative integer. Also note that if $M$ is any non-zero finitely generated module then $G K(M) \geq 1$ and $e(M) \geq 1$, as $A$ is simple infinite-dimensional.

3.2. Somewhat commutative algebras. A somewhat commutative algebra $A$ is an algebra with a finite-dimensional filtration $\mathcal{F}=\left\{A_{i}\right\}_{i \geq 0}$ such that the graded associated algebra is finitely generated commutative ([24, 8.6.9]). Affine somewhat commutative algebras are Noetherian. Then we have from [24, Corol. 8.6.20]:

Corollary 3.2. Any simple somewhat commutative algebra is an algebra with multiplicity.

Example 3.3. The Weyl algebra $A_{n}(\mathrm{k})$ with the Bernstein filtration is a simple somewhat commutative algebra.

Example 3.4. The ring of differential operators $\mathcal{D}(X)$ on an affine smooth algebraic variety $X$ is a simple somewhat commutative algebra; or, more generally, $\mathcal{D}(B)$ the ring of differential operators on an affine regular commutative domain (cf. [15.1.21, 15.3.7, 15.5.6] [24], [8, Section 5]).

3.3. Filtered semi-commutative algebras. A filtered semi-commutative algebra $A$ is a finitely generated algebra with a finite-dimensional filtration $\mathcal{F}=\left\{A_{i}\right\}_{i \geq 0}$ such that the graded associated algebra is semi-commutative ([22]) i.e., generated by elements $x_{1}, \ldots, x_{n}$ with relations $x_{i} x_{j}=\lambda_{i j} x_{j} x_{i}$, for some $0 \neq \lambda_{i j} \in \mathrm{k}$.

Example 3.5. Examples of such algebras include $\mathcal{O}_{q}\left(\mathbb{M}_{n}(\mathrm{k})\right), \mathcal{O}_{q}\left(G L_{n}(\mathrm{k})\right)$, $\mathcal{O}_{q}\left(S L_{n}(\mathrm{k})\right)$ and $U_{q}\left(s l_{n}\right)$, where $0 \neq q$ is not a root of unity (22]).

We have

Proposition 3.6. 22, Theorem 3.8] Any simple filtered semi-commutative algebra is an algebra with multiplicity. 
3.4. Generalized Weyl algebras. We now introduce our main example of algebras with multiplicities.

Let $D$ be an algebra over k. Let $a=\left(a_{1}, \ldots, a_{n}\right)$ be a n-uple of elements of $Z(D)$. Let $\sigma=\left(\sigma_{1}, \ldots, \sigma_{n}\right)$ be a tuple of automorphisms of $D$ such that $\sigma_{i} \sigma_{j}=\sigma_{j} \sigma_{i}$, $\sigma_{i}\left(a_{j}\right)=a_{j}$, if $j \neq i$. The generalized Weyl algebra (GWA for short) of rank $n$ ([2]) is the algebra with generators $D, X_{i}, Y_{i}, i=1, \ldots, n$, and relations

$$
\begin{gathered}
X_{i} \lambda=\sigma_{i}(\lambda) X_{i} ; Y_{i} \lambda=\sigma_{i}^{-1}(\lambda) Y_{i}, \lambda \in D \\
Y_{i} X_{i}=a_{i}, X_{i} Y_{i}=\sigma_{i}\left(a_{i}\right)
\end{gathered}
$$

We will denote this algebra by $D(a, \sigma)$ and call $X_{i}, Y_{i}$ 's the GWA generators, $D$ the defining algebra. We will assume that $D$ is an affine commutative domain. In this case every generalized Weyl algebra is a Noetherian domain and, hence, an Ore domain. The tensor product over k of two generalized Weyl algebras $D(a, \sigma) \otimes$ $D\left(a^{\prime}, \sigma^{\prime}\right) \simeq\left(D \otimes D^{\prime}\right)\left(a * a^{\prime}, \sigma * \sigma^{\prime}\right)$, is again a generalized Weyl algebra, where $*$ is the tensor product of automorphisms, and the concatenation of $a, a^{\prime}$. In particular we have

Definition 3.7 (2]). Let $D(a, \sigma)$ be a GWA of rank 1, with automorphism $\sigma$ of infinite order. We define $D_{n}(a, \sigma)$ to be $D(a, \sigma)^{\otimes n}$. It is itself a $G W A D^{\prime}(a=$ $\left.\left(a_{1}, \ldots, a_{n}\right), \sigma=\left(\sigma_{1}, \ldots, \sigma_{n}\right)\right)$, where $D^{\prime}=D \otimes \ldots \otimes D n$ times, $a_{i}=1 \otimes \ldots \otimes a \otimes$ $\ldots 1, a$ in the $i$-th position, and $\sigma_{i}=1 \otimes \ldots \otimes \sigma \otimes \ldots 1, \sigma$ in the $i$-th position.

Following 11 we introduce generalized Weyl algebras of classical and of quantum types. Let $A=\mathrm{k}[h](a, \sigma)$ be a generalized Weyl algebra of rank 1 with $\sigma(h)=h-1$. Assume that there is no irreducible polynomial $p \in \mathrm{k}[x]$ such that both $p, \sigma^{i}(p)$ are multiples of $a$ for any $i \geq 0$. Then $A$ is a generalized Weyl algebra of rank 1 of simple classical type. Examples of such algebras are the Weyl algebras, certain subalgebras of their invariants, and certain primitive quotients of $U\left(s l_{2}\right)$, among others (cf. [11, Section 2]).

If $A=\mathrm{k}\left[h^{ \pm}\right](a, \sigma)$ is a generalized Weyl algebra of rank 1 with $\sigma(h)=\lambda h$, $0,1 \neq \lambda \in \mathrm{k}, a \in \mathrm{k}[h], \lambda$ is not a root of unity and there are no irreducible polynomials $p \in \mathrm{k}[x]$ such that both $p, \sigma^{i}(p)$ are multiples of $a$ for any $i \geq 0$, then $A$ is of simple quantum type. Examples of such algebras are certain primitive quotients of $U_{q}\left(s l_{2}\right)$, the quantum torus (cf. [11, Section 2]).

Both kinds of algebras are simple.

Let $A$ be a generalized Weyl algebra of rank 1 of simple classical or quantum type, $X, Y$ the $G W A$ generators, i.e., $X Y=h$. Let $m=\operatorname{deg} a$ and $\alpha$ its leading coefficient. Define a finite-dimensional filtration by $\mathcal{B}_{A}=\left\{B_{i}\right\}_{i>0}$, $B_{n}=\operatorname{span}\left\langle H^{i} v_{j}\right\rangle, 2|i|+m|j| \leq n$, where $v_{j}=X^{j}$ if $j>0, v_{j}=Y^{j}$ if $j<0, v_{0}=1$. Let $A=\otimes_{j=0}^{s} A_{j}$ be a generalized Weyl algebra of rank $s$, where $A_{j}$ are generalized Weyl algebras of simple classical or quantum types. Consider the tensor product of the filtrations $\mathcal{B}_{A}=\otimes_{j=0}^{s} \mathcal{B}_{A_{j}}$. Then $\mathcal{B}_{A}$ is a finite-dimensional filtration.

Definition 3.8. A generalized Weyl algebra A of rank $s$ which is the tensor product of generalized Weyl algebras of simple quantum and classical type is called a generalized Weyl algebra of mixed type. If all factors of the tensor product are of the same simple type then the algebra $A$ is called a generalized Weyl algebra of pure type. They are simple algebras. 
We have

Theorem 3.9. Let $A$ be a generalized Weyl algebra of rank s.

- If $A$ if mixed type then $G K(A)=2 s$. In this case for each finitely generated $A$-module $M$ we have $G K(M) \geq s$.

- If $A$ is of pure type then $A$ is algebra with multiplicity.

Proof. The claim about generalized Weyl algebras of mixed type follows from [11, Theorem 2.1]. For generalized Weyl algebras of pure type, the filtration above shows that it is either somewhat commutative (the case of all tensor factors of simple classical type); or else filtered semi-commutative after taking a quotient by normal elements in the graded associated algebra (the case of all tensor factors of simple quantum type), as shown in [11, Section 2] (cf. [3, Theorem 2.2, 3.2]).

3.5. Category $\mathcal{H}$. For an algebra with multiplicity $A$, denote by $\mathcal{H}=\mathcal{H}(A)$ the category of finitely generated holonomic modules, that is for any $M \in \mathcal{H}, G K(M)=$ $h_{A}$.

Theorem 3.10. The category $\mathcal{H}$ is abelian and every module $M \in \mathcal{H}$ has finite length bounded by $e(M)$. Finally, if $A$ is not Artinian, every holonomic module is cyclic.

Proof. First statement is clear. Let $M \in \mathcal{H}, M=M_{0} \supset M_{1} \ldots \supset M_{s}$ be a strictly descending chain of submodules. Hence $M_{i} \in \mathcal{H}$ and $M_{i} / M_{i+1} \in \mathcal{H}$. As $e\left(M_{i} / M_{i+1}\right) \geq 1$, then we have

$$
s \leq \sum_{j=0}^{s-1} e\left(M_{j} / M_{j+1}\right)=e\left(M / M_{s}\right) \leq e(M) .
$$

Hence, every module $M \in \mathcal{H}$ is Artinian, and since $M$ is finitely generated then $M$ is also Noetherian. Finally, if $A$ is not Artinian, $M$ is cyclic by [17, Theorem $2.5]$.

We immediately have

Corollary 3.11. Every module $M \in \mathcal{H}$ with $e(M)=1$ is irreducible.

We also have

Corollary 3.12. If $G K(A)>h_{A}$, then every module in $\mathcal{H}$ has torsion.

Proof. Let $M \in \mathcal{H}, 0 \neq m \in M$. Consider the map $\phi: A \rightarrow M, a \mapsto a m$. Then $\operatorname{Im} \phi$ is a non-null submodule of $M$, and hence by Theorem 3.10 , belongs to $\mathcal{H}$. Consider the short exact sequence $0 \rightarrow \operatorname{ker} \phi \rightarrow A \rightarrow \operatorname{Im} \phi \rightarrow 0$. $G K(A)=$ $\max \{G K(\operatorname{ker} \phi), G K(\operatorname{Im} \phi)\}$, so $G K(\operatorname{ker} \phi)=G K(A)>0$ as $G K \operatorname{ker}(\phi)=h_{A}$; this implies that the kernel is nonzero and hence $M$ has a torsion.

On the other hand the following is valid.

Proposition 3.13. Let $A$ be a domain with multiplicity and $I$ a non-zero left ideal of $A$. Then $G K(A / I) \leq G K(A)-1$.

Proof. Suppose first that $I=A a, 0 \neq a \in A$. Consider a short exact sequence

$$
0 \rightarrow A \rightarrow A \rightarrow A / A a \rightarrow 0
$$


where the first map is a multiplication by $a$. If $G K(A / A a)=G K(A)$, then $m(A)=$ $m(A)+m(A / A a)>m(A)$ which is clearly absurd. Hence, $G K(A / A a)<G K(A)$. In the general case, $I$ contains a principal left ideal $A a$, and $A / I$ is a quotient of $A / A a$. Hence $G K(A / I)<G K(A)$.

Theorem 3.14. Let $A$ be a domain with multiplicity such that $G K(A)=2$ and $h_{A}=1$. Then a finitely generated $A$-module $M$ belongs to $\mathcal{H}$ if and only if it has a torsion.

Proof. Assume that the module $M$ has a torsion. Let us show that $M$ is holonomic. Suppose that $M$ is generated by elements $m_{1}, \ldots, m_{s}$. Since $M$ has a torsion, $A m_{i}$ is a quotient of $A / J_{i}$ for certain left ideals $0 \neq J_{i} \subset A, i=1, \ldots, s$. Each $A / J_{i}$ is holonomic by the proposition above with the Gelfand-Kirillov dimension 1 . Hence all modules $A m_{i}$ and $M=\sum_{i=0}^{s} A m_{i}$ are holonomic by Theorem 3.10 .

As a consequence we immediately obtain the following well-known result

Corollary 3.15. Let $\mathrm{k}$ be algebraically closed. Every finitely generated weight module for $A_{n}(\mathrm{k})$ is holonomic.

Proof. Every finitely generated weight $A_{n}(\mathrm{k})$-module $M$ has finite length. Since $A_{n}(\mathrm{k})$ is an algebra with multiplicity, it suffices to consider the case when $M$ is a simple module. In this case we have that $M$ is the tensor product of $n$-simple weight modules for $A_{1}(\mathrm{k})$ (cf. 7]). But every such $A_{1}(\mathrm{k})$-module has a torsion. Hence, the result follows from Theorem 3.14 .

\section{Filter dimension of invariants}

Assume that char $\mathrm{k}=0$ and $A$ is a simple finitely generated Noetherian algebra. Let $a_{1}, \ldots a_{n}$ be the generated of $A$.

Define a filtration $\mathcal{F}=\left\{A_{i}\right\}_{i \geq 0}: A_{0}=\mathrm{k}, A_{1}=\operatorname{span}\left\langle 1, a_{1}, \ldots, a_{n}\right\rangle, A_{i}=A_{1}^{i}$, $i=1, \ldots, n$. Set

$$
\nu_{\mathcal{F}}(i)=\inf \left\{j \in \mathbb{N} \cup\{\infty\} \mid 1 \in A_{j} a A_{j}, \forall a \in A_{i}\right\} .
$$

Proposition 4.1 ([11, Lemma 1.1). $\Gamma\left(\nu_{\mathcal{F}}\right)=f \operatorname{dim} A$.

We recall some facts on the invariants of noncommutative rings that will be used in what follows (cf. [25, Theorem 2.5, Corollary 2.6], [26] and [24, 8.2.9]).

Theorem 4.2. Let $G$ be a finite group of outer automorphisms of $A$ and $A * G$ the skew group ring.

(1) $A^{G}$ is a simple ring Morita equivalent to $A * G$ and $G K\left(A^{G}\right)=G K(A)$.

(2) $A^{G}$ is finitely generated and Noetherian; if $A$ is not Artinian then neither is $A^{G}$.

We are now going to explore the connection between the filter dimensions of $A$ and $A^{G}$. Suppose that $G$ acts by outer automorphisms. We can assume without loss of generality that $G$ stabilizes $A_{1}$ : just consider the set $\left\{g\left(a_{i}\right) \mid g \in G, i=1, \ldots, n\right\}$ as generators for $A$. Then the algebra $A * G$ is simple by Theorem 4.2, and posseses a filtration $\mathcal{F}^{\prime}=\left\{B_{i}\right\}_{i \geq 0}$, with $B_{0}=\mathrm{k}$ and $B_{1}=\operatorname{span}\left\langle a_{1}, \ldots, a_{n}, g \in G\right\rangle$.

Lemma 4.3. For every $i, \nu_{\mathcal{F}^{\prime}}(i) \leq \nu_{\mathcal{F}}(i)$. 
Proof. Let $b \in B_{i}$. If $\nu_{\mathcal{F}}(i)=\infty$ then the claim is clear. Otherwise, consider the idempotent $e=\frac{1}{|G|} \sum_{g \in G} g$. Then $e \in B_{j}, j \geq 1$. We also have $A_{j} \subset B_{j}$. Symmetrizing we have ebe $\in A^{G} \subset A$; in fact ebe $\in A_{j}$. Hence, if $\nu_{\mathcal{F}}(i)=k$ then $1 \in A_{k}$ ebe $A_{k}$, and hence $1 \in B_{k} b B_{k}$. So we are done.

Proposition 4.4. We have $f \operatorname{dim} A^{G} \leq f \operatorname{dim} A$. Moreover, if $f \operatorname{dim} A=1$ and there exists a finitely generated $A^{G}$-module $M$ such that $G K(M) \leq \frac{1}{2} G K\left(A^{G}\right)$, then $f \operatorname{dim} A=f \operatorname{dim} A^{G}$.

Proof. Since $A^{G}$ and $A * G$ are Morita equivalent by Theorem 4.2 (2), then the first claim follows from the above lemma and Theorem 2.4. If $f \operatorname{dim} A=1$ then $f \operatorname{dim} A^{G} \leq 1$. If there exists a module $M$ which satisfying the hypothesis then fdim $A^{G} \geq 1$ by [8, Corollary 1.7(i)]. The statement follows.

\section{Holonomic MOdUles For INVARIANT SUbalgebras}

This section contains our main results. We assume that $\mathrm{k}$ has characteristic 0 and use the theory developed in previous sections to show that $A(\mathrm{k})^{G}$ and $\mathcal{D}\left(\mathbb{A}^{n} / G\right)$ have a good theory of holonomic modules for suitable actions of $G$ (Theorems 5.7. 5.8, 5.9). The same holds for adequate invariant subrings of generalized Weyl algebras of pure type (Theorems 5.11 5.12).

5.1. Invariant differential operators. Let $A$ be a commutative k-algebra. The algebra of differential operators on $A$ is defined as follows. Set $\mathcal{D}(A)_{0}=A, \mathcal{D}(A)_{n}=$ $\left\{d \in \operatorname{End}_{\mathrm{k}} A \mid[d, a] \in \mathcal{D}(A)_{n-1}, \forall a \in A\right\}$, and $\mathcal{D}(A)=\bigcup_{i=0}^{\infty} \mathcal{D}(A)_{i}$. This way we obtain a natural structure of filtered associative k-algebra.

We recall

Proposition 5.1. 24, Chapter 15] If $A$ is affine and regular then $\mathcal{D}(A)$ coincides with the subring of End $d_{\mathrm{k}} A$ generated by $A$ and the module $D e r_{\mathrm{k}} A$ of $\mathrm{k}$-derivations. $\mathcal{D}(A)$ is a simple affine Noetherian domain, and $G K(\mathcal{D}(A))=2 G K(A)$.

Through the rest of this subsection we assume that $A$ is an affine regular commutative domain with finite Krull dimension, i.e. the algebra of regular functions on a smooth affine irreducible variety. Then the algebra $\mathcal{D}(A)$ has a finite-dimensional filtration $\mathcal{F}$ such that $g r_{\mathcal{F}} \mathcal{D}(A)$ is affine commutative by [24, 15.1.21, 15.3.7,15.5.6], that is $\mathcal{D}(A)$ is a somewhat commutative algebra (cf. Example 3.4). In particular, $\mathcal{D}(A)$ is a simple algebra with multiplicity and $f \operatorname{dim} \mathcal{D}(A)=1$ (cf. Example 2.2). Note that $\mathcal{D}(A)$ is not an Artinian ring.

Let $G$ be a finite group of algebra automorphisms of $A$. Then $G$ acts on $\mathcal{D}(A)$ by conjugation: if $g \in G$ and $d \in \mathcal{D}(A)$ then $g . d=g d g^{-1}$. The subalgebra $\mathcal{D}(A)^{G}$ of $G$-invariant differential operators inherits a finite-dimensional filtration $\mathcal{F}^{\prime}=$ $\left\{A_{i}^{\prime}:=A^{G} \cap A_{i}\right\}_{i \geq 0}$.

Proposition 5.2. $\mathcal{D}(A)^{G}$ is a somewhat commutative algebra with the filtration $\mathcal{F}^{\prime}$.

Proof. Since $A$ and $\operatorname{Der}_{\mathrm{k}} A$ are $G$-stable then without loss of generality one can assume that for each $A_{i}, i \geq 0$ in the filtration $\mathcal{F}$ holds $G\left(A_{i}\right) \subset A_{i}, i \geq 0$. This is due to [24, Proposition 8.6.7, 8.6.9] and to the fact that $\mathcal{D}(A)$ is an almost centralizing extension of $A$ [24, Theorem 15.1.20(i)]. Then we have that 
$g r_{\mathcal{F}^{\prime}} \mathcal{D}(A)^{G} \simeq\left(g r_{\mathcal{F}} \mathcal{D}(A)\right)^{G}$ by [18, 3.2.3]. By the Noether's Theorem, the right hand side of this isomorphism is an affine algebra and the statement follows.

Proposition 5.3. The units of $\mathcal{D}(A)$ are the units of $A$.

Proof. Let $y_{1}, \ldots, y_{t}$ be a transcendence basis of the field of fractions of $A$. Suppose that $x \in \mathcal{D}(A)$ is a unit. A localization $\mathcal{D}(A)_{c}$ of $\mathcal{D}(A)$ by a certain non-zero regular element $c$ is isomorphic to an iterated Ore extension $A\left[x_{1} ;-\partial_{y_{1}}\right], \ldots,\left[x_{t} ;-\partial_{y_{t}}\right]$ of $A$, by [24, 15.1.25, 15.2.6, 15.3.2]. Since $\mathcal{D}(A)$ embeds into $\mathcal{D}(A)_{c}$, then $x$ is a unit of $A\left[x_{1} ;-\partial_{y_{1}}\right], \ldots,\left[x_{t} ;-\partial_{y_{t}}\right]$, and hence of $A$.

From Proposition 5.3 we immediately have

Corollary 5.4. Let $G$ be a non-trivial finite group of automorphisms of $A$ with induced action on $\mathcal{D}(A)$ by conjugation. Then the action of $G$ is outer.

Theorem 5.5. Let $G$ be a finite group of automorphisms of $A$ with induced action on $\mathcal{D}(A)$ by conjugation. Set $n=G K(A)$. Then

(1) $\mathcal{D}(A)^{G}$ is a simple ring Morita equivalent to $\mathcal{D}(A) * G$, both have the Gelfand-Kirillov dimension 2 .

(2) $\mathcal{D}(A)^{G}$ is finitely generated Noetherian but not Artinian.

(3) $f \operatorname{dim} \mathcal{D}(A)^{G}=1, h_{\mathcal{D}(A)^{G}}=n$, and every finitely generated $\mathcal{D}(A)^{G}$ module $M$ satisfies the Bernstein inequality $G K(M) \geq n$.

(4) Let $M \in \mathcal{H}\left(\mathcal{D}(A)^{G}\right)$ be a holonomic $\mathcal{D}(A)^{G}$-module. Then $M$ is cyclic torsion module with finite length bounded by $e(M)$. Moreover, $M$ is simple if $e(M)=1$.

Proof. Applying Theorem 4.2, Proposition 5.1 and Corollary 5.4 we obtain first two statements. Recall that $f \operatorname{dim} \mathcal{D}(A)=1$. We have that $A^{G}$ is a $\mathcal{D}(A)^{G}$-module of the Gelfand-Kirillov dimension $n$. Hence, by Proposition 4.4 $f \operatorname{dim} \mathcal{D}(A)^{G}=1$. The Bernstein inequality then follows from Theorem 2.3 . Since we have explicitly constructed a module with the minimal possible Gelfand-Kirillov dimension, then $h_{\mathcal{D}(A)^{G}}=n$.

5.2. Invariants of the Weyl algebra. Let $x_{1}, \ldots, x_{n}, y_{1}, \ldots, y_{n}$ be the standard generators of the Weyl algebra $A_{n}(\mathrm{k})$, identified with the algebra of $\mathcal{D}\left(\mathrm{k}\left[x_{1}, \ldots, x_{n}\right]\right)$ of differential operators on the polynomial ring. The linear actions of finite groups of automorphisms on $A_{n}(\mathrm{k})$ by conjugation are induced from the linear actions on $\mathrm{k}\left[x_{1}, \ldots, x_{n}\right]$. Recall that an automorphism of $A_{n}(\mathrm{k})$ that fixes the subspace $\operatorname{span}\left\langle x_{1}, \ldots, x_{n}, y_{1}, \ldots, y_{n}\right\rangle$ is called a symplectic automorphism. In particular, every linear automorphism of $A_{n}(\mathrm{k})$ is a symplectic automorphism [18].

Corollary 5.6. Let $G$ be a finite group of symplectic automorphisms of $A_{n}(\mathbf{k})$. We have:

(1) $A_{n}(\mathrm{k})^{G}$ is a simple ring Morita equivalent to $A_{n}(\mathrm{k}) * G$, both have the Gelfand-Kirillov dimension $2 n$.

(2) $A_{n}(\mathrm{k})^{G}$ is finitely generated Noetherian but not Artinian.

(3) $A_{n}(\mathrm{k})^{G}$ is a somewhat commutative algebra.

Proof. Statements (1) and (2) follow immediately from Theorem 4.2. Introduce a filtration $\mathcal{E}=\left\{E_{i}\right\}_{i \geq 0}$ on $A_{n}(\mathrm{k})^{G}$ induced from the Bernstein filtration $\mathcal{B}=$ $\left\{B_{i}\right\}_{i \geq 0}: E_{i}=B_{i} \cap A_{n}(\mathrm{k})^{G}$. Then $g r_{\mathcal{E}} A_{n}(\mathrm{k})^{G} \simeq\left(g r_{\mathcal{B}} A_{n}(\mathrm{k})\right)^{G}([18])$, which is a 
finitely generated commutative algebra by the Noether's theorem. Statement (3) follows.

Theorem 5.7. Let $G$ be a finite group of symplectic automorphisms of $A_{n}(\mathrm{k})$.

- $\operatorname{fdim} A_{n}(\mathrm{k})^{G}=1, h_{A_{n}(\mathrm{k})^{G}}=n$, and every finitely generated $A_{n}(\mathrm{k})^{G}$ module $M$ satisfies the Bernstein inequality $G K(M) \geq n$.

- If $M$ is a holonomic $A_{n}(\mathrm{k})$-module then $M$ is a cyclic torsion module of finite length bounded by $e(M)$. In particular, $M$ is simple if $e(M)=1$.

Proof. If the action of $G$ is linear then the statements follow from Theorem 5.5 . We now consider an arbitrary action of $G$. First note that $G$ preserves the Bernstein filtration of $A_{n}(\mathrm{k})$. Since $G$ is finite then we have $h_{A_{n}(\mathrm{k}) * G}=h_{A_{n}(\mathrm{k})}=n$. Hence $h_{A^{G}}=n$ by Theorem 2.4. Since the algebra $A_{n}(\mathrm{k})^{G}$ is somewhat commutative, there exists a module $0 \neq M$ with the minimal Gelfand-Kirillov dimension. Hence, $\operatorname{fdim} A_{n}(\mathrm{k})^{G}=1$ by Proposition 4.4

5.3. Differential operators on quotient varieties. From now on we assume that $\mathrm{k}$ is algebraically closed. Consider the quotient of $\mathbb{A}^{n}=S p e c \mathrm{k}\left[x_{1}, \ldots, x_{n}\right]$ by $G$, and the ring of differential operators on $\mathbb{A}^{n} / G$. Note that in general $\mathbb{A}^{n} / G$ is a singular variety, except when $G$ is a pseudo-reflection group by the ChevalleyShephard-Todd theorem ([14, Theorem 7.2.1]).

Let $W$ be a finite group of linear automorphisms of $\mathrm{k}\left[x_{1}, \ldots, x_{n}\right], N \subset W$ a subgroup generated by the pseudoreflections. Then $N$ is normal in $W$ (cf. 28, pp. 259]). Further on, we have natural isomorphisms

$$
\mathrm{k}\left[x_{1}, \ldots, x_{n}\right]^{W} \simeq\left(\mathrm{k}\left[x_{1}, \ldots, x_{n}\right]^{N}\right)^{W / N} \simeq \mathrm{k}\left[x_{1}, \ldots, x_{n}\right]^{W / N},
$$

where the second isomorphism follows from the Chevalley-Shephard-Todd theorem. Moreover, the induced action of $W / N$ on the polynomial algebra is linear. Now we have

Lemma 5.8. Let $W$ be any finite group of linear automorphisms of the polynomial ring $\mathrm{k}\left[x_{1}, \ldots, x_{n}\right]$ (and hence of the affine space $\mathbb{A}^{n}$ ). Then $\mathcal{D}\left(\mathbb{A}^{n} / W\right.$ ) is isomorphic to the ring of invariants of $A_{n}(\mathrm{k})$ under the action of a finite group of linear automorphisms.

Proof. Since $W / N$ does not contain pseudoreflections then using the isomorphisms above and [21, Theorem 5] we have an isomorphism of algebras of invariant differential operators

$$
\mathcal{D}\left(\mathrm{k}\left[x_{1}, \ldots, x_{n}\right]^{N}\right)^{W / N} \simeq \mathcal{D}\left(\mathrm{k}\left[x_{1}, \ldots, x_{n}\right]^{W}\right) .
$$

Since $W / N$ acts linearly on

$$
\mathrm{k}\left[x_{1}, \ldots, x_{n}\right]^{N} \simeq \mathrm{k}\left[x_{1}, \ldots, x_{n}\right]
$$

we have $\mathcal{D}\left(\mathbb{A}^{n} / W\right) \simeq \mathcal{D}\left(\mathbb{A}^{n}\right)^{W^{\prime}}$ with linear action of $W^{\prime} \simeq W / N$.

From Lemma 5.8 and Theorem 5.7 we immediately have

Theorem 5.9. Let $W$ be a finite group of linear automorphisms of $\mathbb{A}^{n}$.

- The ring $\mathcal{D}\left(\mathbb{A}^{n} / W\right)$ has the filter dimension 1 ; 
- If $M$ is a holonomic $\mathcal{D}\left(\mathbb{A}^{n} / W\right)$-module then $M$ is cyclic torsion module with finite length bounded by $e(M)$. In particular, if $e(M)=1$ then $M$ is simple.

5.4. Invariants of generalized Weyl algebras of pure type. We keep the hypothesis that $k$ is algebraically closed. Let us recall the definition of the ShephardTodd groups of type $G(m, p, n)$. Let $G_{m} \subset \mathrm{k}$ be the cyclic group in $m$ elements, generated by the $m$-th roots of unity. Let $A(m, p, n)$ be the subgroup of $G_{m}^{\otimes n}$ consisting of $\left(h_{1}, \ldots, h_{n}\right)$ such that $\left(\prod h_{i}\right)^{m / p}=1$. Set $G(m, p, n)=A(m, p, n) \rtimes S_{n}$. It is always a normal subgroup of $G(m, 1, n)$, and the quotient group is isomorphic to $G_{p}$. The groups of type $G(m, p, n)$ are the non-exceptional irreducible complex reflection groups in the classification of Shephard-Todd.

The following is clear.

Proposition 5.10. Let $A=D(a, \sigma)$ be a generalized Weyl algebra of rank $n$ of pure type, so that $D=k\left[h_{1}, \ldots, h_{n}\right]$ or $\mathrm{k}\left[h_{1}^{ \pm}, \ldots, h_{n}^{ \pm}\right]$. Then $A$ is equipped with the following natural action of $G(m, p, n)$ : if $\xi=(g, \pi) \in A(m, p, n) \rtimes S_{n}, g=$ $\left(g_{1}, \ldots, g_{n}\right)$, then $\xi\left(h_{i}\right)=h_{\pi(i)}, \xi X_{i}=g_{i} X_{\pi(i)}, \xi Y_{i}=g_{i}^{-1} Y_{\pi(i)}$.

We have

Theorem 5.11. Let $A=D(a, \sigma)$ be a generalized Weyl algebra of rank $n$ of pure type, and $G=G(m, p, n)$. Then we have:

- $A^{G}$ is a finitely generated simple Noetherian ring which is not Artinian; $A^{G}$ is Morita equivalent to $A * G$.

- For every finitely generated $A^{G}$-module $M, G K(M) \geq n$.

- $f \operatorname{dim} A^{G}=1$.

Proof. Since $G=G(m, p, n)$ acts by outer automorphisms on $A$, we are in the position to apply Theorem 4.2 and Proposition 4.4. Item (1) follows by the same proof as of Corollary [5.6. Since $G$ preserves the filtration $\mathcal{B}_{A}$, it is clear that $h_{A * G}=h_{A}=n$. Hence the statement (2) follows from Theorem 2.4. Finally, Statement (3) follows from Proposition 4.4

Finally, consider the category of holonomic modules $\mathcal{H}(A)$ for $A=D(a, \sigma)^{G(m, p, n)}$.

Theorem 5.12. Let $A=D(a, \sigma)^{G(m, p, n)}$ be the invariant subalgebra of a generalized Weyl algebra of rank $n$ of pure type. If $M \in \mathcal{H}(A)$ then $M$ is a cyclic torsion module with finite length bounded by $e(M)$. If $e(M)=1$ then $M$ is simple.

Let us now revisit Theorem 3.14. We have

Theorem 5.13. Let $A$ be one of the following algebras:

(1) Any subalgebra of invariants of $A_{1}(\mathbb{C})$ under the action of a finite group;

(2) The ring of differential operators $\mathcal{D}(X)$, where $X$ is an smooth affine curve;

(3) A generalized Weyl algebra of rank 1 of simple classical or quantum type.

If $M$ is a finitely generated $A$-module then $G K(M)=1$ if and only if $M$ has a torsion.

Proof. Let $G$ be any finite group of automorphisms of $A_{1}(\mathbb{C})$. Then $G$ is conjugated in $A u t_{\mathbb{C}} A_{1}(\mathbb{C})$ to a group of symplectic automorphisms [1 (cf. [18). Now the statement in the case of the first Weyl algebra follows from Theorem 3.14 and 
Theorem 5.7. The case of differential operators on curves follows from Theorem 3.14 and Example 3.4. The case of generalized Weyl algebras follows from Theorem 3.14 and Theorem 3.9 .

\section{Rational Cherednik algebras}

6.0.1. Generalized somewhat commutative algebras. Let $W$ be a complex reflection group acting on a complex vector space $H, S$ the set of reflections. For each $s \in S$, take $\alpha_{s} \in H^{*}$ and $\alpha_{s}^{\vee} \in H$ such that $\alpha_{s}$ is an eigenvector of $\lambda_{s}$ (the non-trivial eigenvalue of $s$ in $h^{*}$ ); and $\alpha_{s}^{\vee}$ is an eigenvector of $\lambda_{s}^{-1}$ (the non-trivial eigenvalue of $s$ in $H)$. Normalize them in such a way that $\left(\alpha_{s}, \alpha_{s}^{\vee}\right)=2$ with respect to the natural pairing $H^{*} \times H \rightarrow \mathbb{C}$. Finally, let $c: S \rightarrow \mathbb{C}$ be the invariant conjugation function.

The rational Cherednik algebra $H_{c, t}(W, H), t \in \mathbb{C}$ is the quotient of $\mathbb{C} W \ltimes T(H \oplus$ $\left.H^{*}\right)$ by the relations:

$$
\left[x, x^{\prime}\right]=\left[y, y^{\prime}\right]=0 ;[y, x]=t x(y)-\sum_{s \in S} c(s)\left(y, \alpha_{s}\right)\left(x, \alpha_{s}^{\vee}\right) s,
$$

with $x, x^{\prime} \in H^{*}, y, y^{\prime} \in H$.

We also consider the spherical subalgebra $U_{c, t}(W, H):=e H_{c, t}(W, H) e$ of $H_{c, t}(W, H)$, where $e:=\frac{1}{|W|} \sum_{w \in W} w$.

Without loss of generality we assume $t=1$ and for simplicity just write $H_{c}$ and $U_{c}$ for the algebras above, and $\mathrm{n}$ for $\operatorname{dim} H$. Recall that for a generic $c$, both algebras are Morita equivalent, simple Noetherian but not Artinian rings (cf. [27]).

Let us call an algebra $A$ generalized somewhat commutative algebra if it has a finite filtration $\mathcal{F}=\left\{A_{i}\right\}_{i \geq 0}$ with $\mathrm{k} \subset A_{0}$ and $\operatorname{dim} A_{i}<\infty, i \geq 0$, such that the associated graded algebra is affine commutative. The difference between this definition and the definition of somewhat commutative algebra is that we do not impose a condition $A_{0}=\mathrm{k}$. Nonetheless, we have:

Theorem 6.1. Let $A$ is an affine simple generalized somewhat commutative algebra. Then $A$ is an algebra with multiplicity.

Proof. It follows from [3, Theorem 3.2, Proposition 3.3], using the fact the dimension defined there equals to the Gelfand-Kirillov dimension by 20, Lemma 2.1, Proposition 6.6].

Corollary 6.2. For a generic $c$, the spherical subalgebra $U_{c}$ is a generalized somewhat commutative algebra with multiplicity. Moreover, $G K\left(H_{c}\right)=G K\left(U_{c}\right)=2 \mathrm{n}$.

Proof. The first statement follows from [13, 1.6], as it shows that for an adequate finite filtration, gr $U_{c} \simeq \mathbb{C}\left[h \oplus h^{*}\right]^{W}$. Then the second statement follows from [20, Proposition 6.6] and [24, Propostion 8.2.9(iii)].

6.1. Filter and Krull dimensions of rational Cherednik algebras. We assume that $c$ is a generic parameter.

Lemma 6.3. $f \operatorname{dim} H_{c}=f \operatorname{dim} U_{c} \geq 1$. 
Proof. The Gelfand-Kirillov dimension the polynomial representation of $H_{c}$ equals $\frac{1}{2} G K\left(H_{c}\right)$. Hence, $f \operatorname{dim} H_{c} \geq 1$ by [8, Corollary 1.7(i)]. Since $f d i m$ is Morita invariant, we conclude that $f \operatorname{dim} U_{c} \geq 1$.

Corollary 6.4. If $M \in \mathcal{H}\left(U_{c}\right)$ then $M$ is a cyclic torsion module with finite length bounded by $e(M)$. Moreover, if $e(M)=1$ then $M$ is simple.

Proof. Follows from Lemma 6.3 Theorem 2.3 and Theorem6.5.

We have

Theorem 6.5. $f \operatorname{dim} H_{c}=f \operatorname{dim} U_{c}=1$.

Proof. The algebra $U_{c}$ is an algebra with multiplicity by Corollary 6.2, Let $h$ be the holonomic number of $U_{c}$. Then there exists a finitely generated $U_{c}$-module $M$ such that $G K(M)=h$. Since the Bernstein's inequality holds for $M$ (cf. [27, Proposition 3.7]), $h=\frac{1}{2} G K\left(U_{c}\right)$. Hence $f \operatorname{dim} U_{c} \leq 1$ by Theorem 2.3 Since $f \operatorname{dim}$ is a Morita invariant by Theorem 2.4 then we have $f \operatorname{dim} U_{c}=f \operatorname{dim} H_{c}=1$ by Lemma 6.3

Next we compute the Krull dimension $\mathcal{K}$ (in the sense of Gabriel-Rentschler, cf. 24. Chapter 6]) of these algebras.

Theorem 6.6. $\mathcal{K}\left(H_{c}\right)=\mathcal{K}\left(U_{c}\right)=\mathrm{n}$.

Proof. Since the Krull dimension is a Morita invariant ([24, Proposition 6.5.1]), it suffices to show it for $U_{c}$. By the Dunkl embedding we have that a localization of the spherical subalgebra is isomorphic to $D\left(h_{r e g} / W\right)$ ([13, Proposition 4.4.1]). By [24, Lemma 6.5.3(iib)], $\mathcal{K}\left(U_{c}\right) \geq \mathcal{K}\left(D\left(h_{\text {reg }} / W\right)\right)$, and the later equals $\mathrm{n}$ by [24, Theorem 15.3.7]. Since $f \operatorname{dim} U_{c}=1$ and $G K\left(U_{c}\right)=2 \mathrm{n}$, by [6, Theorem 1.3], then we have $\mathcal{K}\left(U_{c}\right) \leq \mathrm{n}$. Hence the equality follows.

\section{ACKNowledgments}

V.F. is supported in part by the CNPq (304467/2017-0) and by the Fapesp (2018/23690-6); J.S. is supported by the Fapesp (2018/18146-5).

\section{REFERENCES}

[1] J. Alev, F. Dumas. Invariants du corps de Weyl sous l'action de groupes finis, Commun. Algebra 25 (1997), 1655-1672.

[2] V. Bavula. Generalized Weyl algebras and their representations, Algebra i Analiz 4 (1992) 75-97. English translation: St. Petersburg Math. J. 4 (1993) 71-92.

[3] V. Bavula. Identification of the Hilbert function and Poincaré series, and the dimension of modules over filtered rings. Russian Acad. Sci. Izv. Math., 44:225-246, 1995.

[4] V. Bavula. Filter dimension of algebras and modules, a simplicity criterion for generalized Weyl algebras. Comm. Algebra, 24:1971-1992, 1996.

[5] V. Bavula. Classification of the simple modules of the quantum Weyl algebra and the quantum plane. Quantum groups and quantum spaces (Warsaw, 1995), 193-201, Banach Center Publ., 40, Polish Acad. Sci. Inst. Math., Warsaw, 1997.

[6] V. Bavula. Krull, Gelfand-Kirillov, and filter dimensions of simple affine algebras, J. Algebra 206 (1998), no. 1, 3-39.

[7] V. Bavula, V. Bekkert, V. Futorny. Weight modules for Weyl algebras. Kac-Moody Lie algebras and related topics, 17-42, Contemp. Math., 343, Amer. Math. Soc., Providence, RI, 2004.

[8] V. Bavula. Filter dimension. Handbook of algebra. Vol. 4, 77-105, Handb. Algebr., 4 (ed. M. Hazewinkel), Elsevier/North-Holland, Amsterdam, 2006. 
[9] V. Bavula. Quiver generalized Weyl algebras, skew category algebras and diskew polynomial rings. Math. Comput. Sci. 11 (2017), no. 3-4, 253268.

[10] V. Bavula and V. Hinchcliffe. Morita invariance of the filter dimension and of the inequality of Bernstein. Algebr. Represent. Theory 11 (2008), no. 5, 497-504.

[11] V. Bavula and F. van Oystaeyen. Simple holonomic modules over the second Weyl algebra A2. Adv. Math. 150 (2000), no. 1, 80-116

[12] V. Bavula and F. van Oystaeyen. Simple modules of the Witten-Woronowicz algebra, J. Algebra 271 (2004), no. 2, 827-845.

[13] G. Bellamy. Symplectic reflection algebras. Noncommutative algebraic geometry, 167-238, Math. Sci. Res. Inst. Publ., 64, Cambridge Univ. Press, New York, 2016.

[14] D. J. Benson, Polynomial Invariants of Finite Groups. London Mathematical Society Lecture Notes Series 190 (1993), Cambridge University Press.

[15] I.N. Bernstein. Modules over a ring of differential operators. An investigation of the fundamental solutions of equations with constant coefficients. Funkcional. Anal. i Prilozen. 5 (1971), no. 2, 1-16.

[16] A. Borel. Algebraic D-Modules, Perspectives in Mathematics, Academic Press, 1987.

[17] S.C.Coutinho, A primer of algebraic D-modules. London Mathematical Society Student Texts 33. Cambridge University Press, 1995.

[18] F. Dumas, Noncommutative Invariants. Trabajos de Matemática, Série B, n. 60/2011, Universidade Nacional de Córdoba, Facultad de Matemática, Física y Astronomía, 2011. http://math.univ-bpclermont.fr/f́fumas/fichiers/B-Mat60.pdf

[19] J. C. Jantzen, Einhüllende Algebren halbeinfacher Lie-Algebren. (German) [Enveloping algebras of semisimple Lie algebras] Ergebnisse der Mathematik und ihrer Grenzgebiete (3) [Results in Mathematics and Related Areas (3)], 3. Springer-Verlag, Berlin, 1983. ii+298 pp. ISBN: 3-540-12178-1.

[20] G. R. Krause and T. H. Lenegan. Growth of Algebras and Gelfand-Kirillov Dimension, Graduates Studies in Mathematics 22, American Mathematical Society, revised edition, 2000.

[21] T. Levasseur. Anneaus d'operateurs differentiels. Em Séminaire d'Algebre Paul Dubreil et Marie-Pau le Malliavin, Lecture Notes in Mathematics 867, páginas 157-173, Paris, 1980. Springer-Verlag.

[22] J.C. McConnell. Quantum groups, filtered rings and Gel'fand-Kirillov dimension. Noncommutative ring theory (Athens, OH, 1989), 139-147, Lecture Notes in Math., 1448, Springer, Berlin, 1990.

[23] J.C. McConnell. Representations of solvable Lie algebras V: On the Gelfand-Kirillov dimension of simple modules, J. Algebra 76 (1982), 489-493.

[24] J.C. McConnell and J. C. Robson. Noncommutative noetherian rings, revised edition, Graduate Studies in Mathematics 30, American Mathematical Society, Providence, 2001.

[25] S. Montgomery. Fixed rings of finite automorphism groups of associative rings, Lecture Notes in Mathematics, 818. Springer Berlin Heidelberg, 1980, 126 pp.

[26] S. Montgomery and L. W. Small. Fixed rings of noetherian rings. Bull. London. Math. Soc., 13:33-38, 1981

[27] D. Thompson. Holonomic modules over Cherednik algebras, I. J. Algebra 493 (2018), 150-170.

[28] W. N. Traves. Invariant theory and differential operators. Gröbner bases in symbolic analysis, 245-265, Radon Ser. Comput. Appl. Math., 2, Walter de Gruyter, Berlin, 2007.

(V. Futorny) Instituto de Matematica e Estatistica, Universidade de São Paulo, Caixa POSTAL 66281

São Paulo, CEP 05315-970, Brasil and International Center for Mathematics, SUSTech, Shenzhen, China

E-mail address: futorny@ime.usp.br

(J. Schwarz) Instituto de Matematica e Estatistica, Universidade de SÃo Paulo, Caixa

POSTAL 66281

SÃo PAUlo, CEP 05315-970, Brasil

E-mail address: jfschwarz.0791@gmail.com 